\title{
Management of patients with diabetic macular oedema and good visual acuity: new findings from Protocol V
}

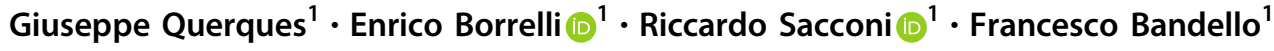

Received: 28 August 2019 / Accepted: 28 August 2019 / Published online: 29 October 2019

(c) The Royal College of Ophthalmologists 2019

As we observe a dramatic increase in diabetes prevalence worldwide, diabetes-associated eye complications are rapidly emerging as a global health issue that may threaten patients' visual acuity [1]. Even though treatment of diabetic retinopathy (DR) can reduce the risk of visual loss by $60 \%$ [2], this disorder still remains the leading cause of blindness among working-age adults.

Diabetic macular oedema (DMO) is a major cause of vision decrease in these patients and may occur at any stage of DR. In 1985, the Early Treatment Diabetic Retinopathy Study reported on the use of laser photocoagulation to treat DMO [3]. The latter trial enrolled 1122 patients with DMO and demonstrated that the laser treatment effects in a reduced risk of moderate vision loss. Until the introduction of intravitreal anti-vascular endothelial growth factor (VEGF) injections, laser had been thus considered as the treatment of choice for eyes with DMO. Since 2010, several evidences have suggested that anti-VEGF agents may be considered as an effective and safe treatment in eyes with DMO and impaired vision [4-8]. To simplify, anti-VEGF therapies demonstrated a better improvement in visual acuity in comparison with focal/grid laser therapy. Nonetheless, the anti-VEGF treatment was also displayed to produce an amelioration in DR severity [9], even without an enhancement in retinal perfusion [10]. Of note, in a number of cases the anti-VEGF treatment may be ineffective and in these cases a switch to other treatments, including intravitreal dexamethasone, was proved to be potentially effective, especially in presence of definite imaging biomarkers [11].

Thanks to the support of the National Eye Institute, National Institutes of Health, the Diabetic Retinopathy Clinical Research (DRCR) Retina Network has organized

Giuseppe Querques

giuseppe.querques@hotmail.it

1 Department of Ophthalmology, University Vita-Salute, IRCCS Ospedale San Raffaele, Milan, Italy and realized different important clinical trials which delineated guidelines for patients with DMO. In detail, in a study on 854 eyes with DMO, they provided evidence that intravitreal ranibizumab is superior in gaining visual acuity, with $30 \%$ of eyes increasing by three lines of visual acuity and $50 \%$ increasing by two lines at 1 year [4]. Successively, the DRCR Retina Network compared the three available anti-VEGF drugs in $660 \mathrm{DMO}$ eyes with moderate to severe visual impairment $[12,13]$. The latter clinical trial demonstrated that all the three agents cause VA improvement from baseline to 1 and 2 years with a decreased number of injections in the second year $[12,13]$. However, aflibercept was displayed to be more efficacious at improving vision at 1 year in eyes with severe visual impairment (20/50 to $20 / 320$ Snellen equivalent) $[12,14]$. Among these eyes with worse baseline visual acuity, aflibercept had superior visual outcomes at 2 years compared with bevacizumab, while superiority of aflibercept over ranibizumab, noted at 1 year, was no longer displayed [13, 14].

Limited data was however available on the most appropriate therapeutic approach for eyes with DMO and good visual acuity. This aspect is crucial, assuming that these patients represent a main portion of DR population [15]. Recently, the DRCR Retina Network investigators reported significant results from Protocol V which specifically sought to address this critical debate [16]. This study included patients with centre-involved DMO and good visual acuity (20/25 or better) who were divided into three arms: prompt laser photocoagulation, prompt aflibercept therapy, or observation. Furthermore, this trial allowed eyes randomized to observation or laser to receive aflibercept rescue if visual acuity decreased from baseline by $\geq 10$ letters at one visit or by 5-9 letters at two following visits [16]. This study concluded that the proportion of eyes experiencing a reduction in visual acuity by five letters at 2 years was similar independently on the assigned group [16]. Moreover, data from this trial also demonstrated that prompt treatment with aflibercept does not cause a significant reduction in the risk of a five letter or more loss, as this 
outcome was reached in 19\%, 17\%, and $16 \%$ in the observation, laser and aflibercept groups, respectively [16]. Importantly, $\sim 10 \%$ of patients within the observation group experienced a two-step improvement in visual acuity, which was similar to that exhibited in the other two groups [16]. Taking all these results together, the investigators concluded that postponing treatment in centre-involved DMO and good visual acuity does not effect in a worse prognosis, as compared with prompt laser or intravitreal treatment.

Assuming that the cost of the drugs would be avoided, these results may have a huge impact on cost and burden of care delivery for patients and the health care system. Nonetheless, a reduction of the psychological burden for patients and their families would also be obtained. Importantly, we might avoid treatment-related unnecessary risks to patients, including the injection procedure itself. All these aspects emphasize the importance of these results and clinicians must recognize their relevance and accordingly employ a conservative management in patients with DMO and good visual acuity, at least until there is recorded reduction in visual acuity.

To completely comprehend these evidences from Protocol $\mathrm{V}$, it is worth noting that included patients in this trial were characterized by a good metabolic and blood pressure control, as well as they routinely attended their follow-up visits. Although it might be said that these subjects do not necessarily reflect the profile of diabetic patients in realworld practice, the OBTAIN study recently reported on real-world data and similarly showed that visual acuity is maintained over a 1 year of follow-up in DMO patients with good visual acuity [17]. However, future studies are needed to reveal whether this conservative management might impact clinic attendance and long-term follow-up care among these patients.

Finally, future developments of more lasting and less invasive therapies might encourage to consider starting treatment earlier. Moreover, further development of novelemerging therapies for DMO [18], including subthreshold laser treatment whose beneficial effect has already been demonstrated [19], may also modify the treatment threshold for these patients. Also, new discovered imaging biomarkers might allow the identification of a sub-group of patients with DMO and good visual acuity who may actually benefit from an early treatment.

\section{Compliance with ethical standards}

Conflict of interest FB is a consultant for: Alcon (Fort Worth,Texas, USA), Alimera Sciences (Alpharetta, Georgia, USA), Allergan Inc (Irvine, California,USA), Bayer Shering-Pharma (Berlin, Germany), Bausch and Lomb (Rochester, New York, USA), Genentech (San Francisco, California, USA), Hoffmann-La-Roche (Basel, Switzerland), NovagaliPharma (Évry, France), Novartis (Basel, Switzerland), Sanofi-Aventis (Paris, France), Thea (Clermont-Ferrand,
France), Thrombogenics (Heverlee,Belgium), and Zeiss (Dublin, USA). GQ is a consultant for: Alimera Sciences (Alpharetta, Georgia, USA), Allergan Inc (Irvine, California, USA), Amgen (Thousand Oaks,USA), Bayer Shering-Pharma (Berlin, Germany), Bausch and Lomb (Rochester, New York, USA), Heidelberg (Germany), KBH (Chengdu; China), Hoffmann-La-Roche (Basel, Switzerland), LEH Pharma (London, UK), Lumithera (Poulsbo; USA), Novartis (Basel, Switzerland), Topcon (Tokyo, Japan), Sandoz (Berlin, Germany), Sifi (Catania, Italy), Sooft-Fidea (Abano, Italy), Thea (Clermont-Ferrand, France), and Zeiss (Dublin, USA). The remaining authors declare that they have no conflict of intetrest.

Publisher's note Springer Nature remains neutral with regard to jurisdictional claims in published maps and institutional affiliations.

\section{References}

1. Querques G. Eye complications of diabetes. Acta Diabetol. 2019;56:971-971. http://www.ncbi.nlm.nih.gov/pubmed/31201573. Accessed 12 Aug 2019.

2. American Diabetes Association AD. Standards of medical care in diabetes-2010. Diabetes Care. 2010;33:S11-61. http://www.ncbi. nlm.nih.gov/pubmed/20042772. Accessed 2 Jun 2019.

3. Photocoagulation for diabetic macular edema: early treatment diabetic retinopathy study report number 1 early treatment diabetic retinopathy study research group. Arch Ophthalmol. 1985;103: 1796-806.

4. Elman MJ, Aiello LP, Beck RW, Bressler NM, Bressler SB, Edwards AR, et al. Randomized trial evaluating ranibizumab plus prompt or deferred laser or triamcinolone plus prompt laser for diabetic macular edema - the diabetic retinopathy clinical research network. Ophthalmology. 2011;118:609-14.

5. Michaelides M, Kaines A, Hamilton RD, Fraser-Bell S, Rajendram R, Quhill F, et al. A prospective randomized trial of intravitreal bevacizumab or laser therapy in the management of diabetic macular edema (BOLT Study). 12-month data: report 2. Ophthalmology. 2010;117:1078-e2.

6. Elman MJ, Bressler NM, Qin H, Beck RW, Ferris FL, Friedman $\mathrm{SM}$, et al. Expanded 2-year follow-up of ranibizumab plus prompt or deferred laser or triamcinolone plus prompt laser for diabetic macular edema. Ophthalmology. 2011;118:609-14.

7. Mitchell P, Bandello F, Schmidt-Erfurth U, Lang GE, Massin P, Schlingemann RO, et al. The RESTORE study: ranibizumab monotherapy or combined with laser versus laser monotherapy for diabetic macular edema. Ophthalmology. 2011;118:615-25.

8. Nguyen QD, Brown DM, Marcus DM, Boyer DS, Patel S, Feiner $\mathrm{L}$, et al. Ranibizumab for diabetic macular edema: Results from 2 phase iii randomized trials: RISE and RIDE. Ophthalmology. 2012;119:789-801.

9. Ip MS, Zhang J, Ehrlich JS. The clinical importance of changes in diabetic retinopathy severity score. Ophthalmology. 2017;124: $596-603$.

10. Bonnin S, Dupas B, Lavia C, Erginay A, Dhundass M, Couturier A, et al. Anti-vascular endothelial growth factor therapy can improve diabetic retinopathy score without change in retinal perfusion. Retina. 2019;39:426-34.

11. Cavalleri M, Cicinelli MV, Parravano M, Varano M, De Geronimo D, Sacconi R, et al. Prognostic role of optical coherence tomography after switch to dexamethasone in diabetic macular edema. Acta Diabetol. 2019: 1-9. http://link.springer.com/10. 1007/s00592-019-01389-4. Accessed 12 Aug 2019.

12. Diabetic Retinopathy Clinical Research Network, Wells JA, Glassman AR, Ayala AR, Jampol LM, Aiello LP, et al. 
Aflibercept, bevacizumab, or ranibizumab for diabetic macular edema. N Engl J Med. 2015;372:1193-203. http://www.ncbi.nlm. nih.gov/pubmed/25692915. Accessed 11 Aug 2019.

13. Wells JA, Glassman AR, Ayala AR, Jampol LM, Bressler NM, Bressler SB, et al. Aflibercept, bevacizumab, or ranibizumab for diabetic macular edema. Ophthalmology. 2016;123:1351-9. https://linkinghub.elsevier.com/retrieve/pii/S0161642016002062. Accessed 11 Aug 2019.

14. Ashraf M, Souka A, Adelman R, Forster SH. Aflibercept in diabetic macular edema: evaluating efficacy as a primary and secondary therapeutic option. Eye. 2016;30:1531-41.

15. Bressler NM, Varma R, Doan QV, Gleeson M, Danese M, Bower $\mathrm{JK}$, et al. Underuse of the health care system by persons with diabetes mellitus and diabetic macular edema in the United States. JAMA Ophthalmol. 2014;132:168-73.

16. Baker CW, Glassman AR, Beaulieu WT, Antoszyk AN, Browning DJ, Chalam KV, et al. Effect of initial management with aflibercept vs laser photocoagulation vs observation on vision loss among patients with diabetic macular edema involving the center of the macula and good visual acuity: a randomized clinical trial. JAMA. 2019;321:1880-94.

17. Busch C, Fraser-Bell S, Zur D, Rodríguez-Valdés PJ, Cebeci Z, Lupidi M, et al. Real-world outcomes of observation and treatment in diabetic macular edema with very good visual acuity: the OBTAIN study. Acta Diabetol. 2019; 56:777-84.

18. Sacconi R, Giuffrè C, Corbelli E, Borrelli E, Querques G, Bandello F. Emerging therapies in the management of macular edema: a review. F1000Research 2019;8:1413 https://f1000resea rch.com/articles/8-1413/v1. Accessed 12 Aug 2019.

19. Mansouri A, Sampat KM, Malik KJ, Steiner JN, Glaser BM. Efficacy of subthreshold micropulse laser in the treatment of diabetic macular edema is influenced by pre-treatment central foveal thickness. Eye. 2014;28:1418-24. 